\title{
EDITORIAL
}

\section{New Perspectives on Indian Agriculture}

\author{
Kamal Bawa* and Kanchan Chopra**
}

The Green Revolution (GR) ushered in an era of unprecedented food security in India. The country not only became self-sufficient in providing food grains to its citizens, but it also started to export them to other countries. However, the intensive agriculture propagated by the GR has also left the land, soils, water, and below and above ground biodiversity severely degraded. The burning of agricultural residue has become a major driver of air pollution, resulting in health hazards during early winters in northern India. This is compounded by additional health risks from water pollution due to the leakage of pesticides and fertilizers into surface and ground water. The impacts of climate change will cause further disruptions to an already unstable system, which continues to generate a significant amount of greenhouse gas emissions, thus perpetuating the problem. This issue of the journal focuses on alternatives to intensive agriculture.

There have indeed already been calls for alternatives to the intensive agriculture approach. Policymakers at the national level have recently called for natural, zero-budget farming. The meaning of the term is not clear, but the rationale is. Other alternative systems include crop diversification, agroecology, climate-smart agriculture, conservation agriculture, regenerative agriculture, and several more. All of these have the same goals: low levels of chemical inputs, improved soil management, and increased below and above ground biodiversity.

Six contributions in this issue deal with contemporary concerns in Indian agriculture, largely in the context of alternatives to intensive agriculture. Raj Paroda comments on how cropping systems need to be diversified to promote greater sustainability and conservation of natural resources. This

\footnotetext{
*ATREE, Bengaluru and University of Massachusetts, Boston. kamal.bawa@gmail.com

${ }^{* *}$ Formerly, Institute of Economic Growth, University of Delhi, Delhi. choprakanchan14@gmail.com
}

Copyright (C) Bawa and Chopra 2022. Released under Creative Commons Attribution (C) NonCommercial 4.0 International licence (CC BY-NC 4.0) by the author.

Published by Indian Society for Ecological Economics (INSEE), c/o Institute of Economic Growth, University Enclave, North Campus, Delhi 110007.

ISSN: 2581-6152 (print); 2581-6101 (web)

DOI: https://doi.org/10.37773/ees.v5i1.661 
paper also suggests possible future crop diversification reforms based on science and suited to specific agro-climatic conditions. These reforms can increase production and farmer incomes and ensure conservation through sustainable intensification.

Incentives will be needed to steer Indian agriculture in a different direction. Ravi Prabhu argues that approaches that follow the principles of agroecology and regenerative agriculture are viable alternatives to industrial agriculture, but incentive-based approaches still depend heavily on the commodification of nature. He presents another alternative, the "stewardship economy", that values the well-being of farmers and other stewards of the land along with rewarding performance in delivering products and services. The transition to this new economy could build on existing reforms and introduce new innovations, resulting in a more resilient and equitable future for Indian agriculture. However, recent backtracking by the government on agricultural reforms in the face of farmers' protests makes one question the practicality of such transitions.

Shannon Olsson and her coauthors point out that regenerative agriculture is gaining attention worldwide as a method to restore and conserve natural resources while maintaining crop productivity. A lack of consensus on what specific conditions define it, however, has made it more difficult for decision-makers, researchers, the agricultural sector, and the public to move forward in adopting these practices. The authors propose a common framework for defining and developing regenerative agriculture practices and techniques in India, considering the viewpoints of multiple stakeholders and the current challenges facing the agricultural sector.

Regenerative agriculture is one of several types of systems that follow agroecological principles. Mihir Shah points out that despite the urgent need to scale up agroecology approaches as alternatives to the GR and its destructive impact, progress in this direction has been slow because the policy framework governing agriculture has remained within a GR paradigm. He suggests key policy reforms that could help remove this impediment to facilitate, support, and accelerate movement towards agroecological farming in India.

Amita Shah and her co-authors highlight the increasing feminization of India's agriculture. They propose that the next phase of agriculture in India will require a deeper understanding of women's contributions as well as of measures to strengthen their role to promote food security across all regions and socio-economic contexts. Based on the results of a survey carried out in Gujarat and West Bengal, the authors discuss the state of food security and the role of women in agriculture in these areas in an 
effort to support the argument that women's roles must be recognized and strengthened by policymakers to promote food security in India.

Kuntala Lahiri-Dutt and Itishree Pattnaik, in a field-based note, further investigate the role (and plight) of farmer households headed by women in the Sundarbans area of West Bengal. The percentage of such households is higher in this part of West Bengal because of the higher incidence of outmigration by men due to limited economic opportunities. Most of the women work as agricultural labourers, are highly indebted, and have limited opportunities to earn income from other sources. Both the agricultural extension services and the government's limited employment scheme, MNREGA, have an extremely limited reach in this part of the state.

Clearly, Indian agriculture faces many challenges. This collection of papers describes and proposes solutions for a few of these challenges. New paradigms and policy frameworks are needed to address the existing and emerging challenges.

The issue carries, in its regular sections, four research papers, four book reviews, and a contributed thematic essay which together reflect the diversity of interests the journal spans. Themes covered in research papers range from "the impact of beliefs on food choices" to the "erosion of biodiversity and culture" and the "valuation of marine ecosystem services". The thematic essay focuses on energy conservation, technological innovations, and behavioral interventions. One cannot help concluding that this first issue of Volume 5 is indeed a weighty one, in ways more than one! 